\title{
Uso de la infografía en la optativa "Alimentación y Dietética" del grado de Medicina
}

\author{
Gloria Olaso González ${ }^{\mathrm{a}}$, Carlos Romá Mateo ${ }^{\mathrm{a}}$, Juan Gambinia, Ángela G. Correas ${ }^{\mathrm{a}}$, Consuelo \\ Escriváa $^{a}$, Marta Piqueras ${ }^{a}$, Consuelo Borrás ${ }^{a}$ y Mari Carmen Gómez-Cabrera ${ }^{a}$. \\ ${ }^{a}$ Departamento de Fisiología, Facultad de Medicina y Odontología, Universidad de Valencia, gloria.olaso@uv.es.
}

\begin{abstract}
As a substitute for the master class, infographics previously designed by the students were used in the session dedicated to treating the characteristics of "fashionable western diets" of the optional subject 34493-Food and Dietetics of the Degree in Medicine of the UV. This initiative was intended to improve student participation in the subject, in addition to deepening the subject, improving their digital skills and the search and synthesis of information. This proposal was well appreciated by the students, who showed their preference for this way of working in front of the master class. In addition, they said that the activity improved their ability to transmit information, to work as a team, their critical and self-critical capacity and that it was very entertaining. From this experience, it could be concluded that infographics are a useful tool for addressing various topics within the Medicine degree.
\end{abstract}

Keywords: methodology, infographics, digital skills, information search, synthesis capacity, medicine, food, diet.

\section{Resumen}

Como sustitución de la clase magistral, en la sesión dedicada a tratar las características de "dietas occidentales de moda" de la asignatura optativa 34493-Alimentación y Dietética del Grado en Medicina de la UV, se utilizaron infografias previamente diseñadas por los estudiantes. Con esta iniciativa se pretendía mejorar la participación del alumnado en la asignatura, además de profundizar en la materia, mejorar sus habilidades digitales y de búsqueda y síntesis de información. Esta propuesta fue bien valorada por el estudiantado, que mostró su preferencia por esta forma de trabajar frente a la clase magistral. Además, opinó que la actividad mejoraba su capacidad de transmitir información, de trabajar en equipo, su capacidad crítica y autocrítica y que resultaba muy entretenida. A partir de esta experiencia se podría concluir que la infografia es una herramienta útil para el abordaje de varios temas dentro del grado de Medicina.

Palabras clave: metodología, infografia, habilidades digitales, búsqueda de información, capacidad de síntesis, medicina, alimentación, dieta.

\section{Introducción}

La optativa Alimentación y Dietética (34493) del Grado en Medicina de la UV, se ofrece en el tercer año de la carrera. Debido a la creciente concienciación sobre la importancia que tiene una alimentación correcta y equilibrada para la salud, la asignatura desde su implantación mantiene todos los cursos un 
número considerable de matriculados (siempre próximo a los 80 ) y además es una de las más demandadas por los estudiantes de la Nau Gran.

La asignatura dedica un tema a las características que debe tener una dieta para ser considerada equilibrada y saludable. Parte del mismo se dedica al análisis de los distintos tipos de dieta más comunes en la sociedad actual y su comparación con los criterios establecidos para definir una dieta equilibrada. El método docente utilizado es la lección magistral, mediante la cual el profesor analiza tres tipos de dietas: dieta mediterránea, dietas vegetarianas y dietas cetogénicas. Este tema interesaba mucho al alumnado, que echaba en falta profundizar más en él y abordar las características de dietas de actual moda entre la población más joven, como es el caso de la dieta macrobiótica, la paleodieta o la dieta "rawfood". Por otra parte, y de modo paradójico, la participación de los estudiantes era baja durante la clase. Optaban por mostrar su interés una vez finalizada la sesión.

La iniciativa que se decidió utilizar de forma alternativa a la clase magistral impartida hasta el momento, consistió en utilizar el diseño de infografías para describir y analizar los distintos tipos de dietas "de moda" en occidente.

Las infografías son capaces de optimizar los procesos de comprensión, ya que se basan en una menor cantidad de texto escrito, concretando mayor información de manera gráfica. Constituyen una forma atractiva y poderosa de generar aprendizaje. Utilizarla como estrategia didáctica tiene como finalidad motivar a los estudiantes y que logren asimilar de manera eficiente los contenidos, capturando su atención.

Con su uso en la asignatura, se pretendió mejorar la participación de los estudiantes y a la vez, profundizar en el análisis de los distintos tipos de dietas comparándolas con los estándares de una dieta equilibrada. Además, los alumnos, mediante la elaboración de las infografías pudieron desarrollar las habilidades de búsqueda, análisis y síntesis de los contenidos y mejorar sus competencias digitales.

Hasta donde sabemos, es la primera vez que se ha utilizado esta herramienta docente en el grado de Medicina de la UV, a pesar de que las infografías sí que se suelen emplear en el ámbito profesional (de la salud (por ejemplo se pueden encontrar muchas a nivel ambulatorio).

\section{Objetivos}

El objetivo principal de esta iniciativa era el de mejorar la participación de los estudiantes en la asignatura y profundizar en el análisis de los distintos tipos de dietas de moda en la sociedad occidental actual. Además, como objetivos secundarios del proyecto, se pretendía desarrollar en los estudiantes habilidades de búsqueda, análisis y síntesis de los contenidos, así como mejorar sus competencias digitales.

\section{Desarrollo de la innovación}

Para realizar esta actividad se utilizaron dos sesiones de clase. En la primera, el docente describió las características de los tres tipos de dietas más importantes: mediterránea, vegetarianas y cetogénicas. A continuación, explicó a los alumnos las características que debe tener una buena infografía. También se mostró el funcionamiento de herramientas gratuitas online (Infogram, Easel.ly, Piktochart, Canva y Create.ly), que facilitan la elaboración de las infografías sin necesidad de tener conocimientos de diseño al ofrecer múltiples plantillas ya predeterminadas. Además, se facilitaron a los alumnos enlaces a dos tutoriales on-line para la realización de infografías con el Powerpoint. En esa misma sesión los alumnos 
se dividieron en 12 grupos, por afinidad, y a cada uno de ellos se les asignó una de las siguientes dietas: Dieta disociada, dieta macrobiótica, dieta Dukan, dieta cruda o "raw-food", dieta Atkins, Dieta paleo, dieta nórdica, dieta de ayuno intermitente, dieta flexitariana, dieta MIND, dieta DASH y dieta DETOX. Asímismo, se les indicó que la información básica que debía facilitar la infografía de cada dieta era la siguiente: 1. Características de la dieta, 2. Distribución de macronutrientes de la dieta, 3. Finalidad de la dieta (terapéutica, perder peso, mantener un estilo de vida saludable, eliminar toxinas, etc.), 4. Población a la que está destinada (población general, con obesidad, con alguna patología...). Se les dio un plazo de 10 días para la búsqueda de información y elaboración de las infografías, que debían enviar al docente. En la Figura 1 se puede observar un esquema con los pasos del proceso de elaboración.

\section{Metodología}

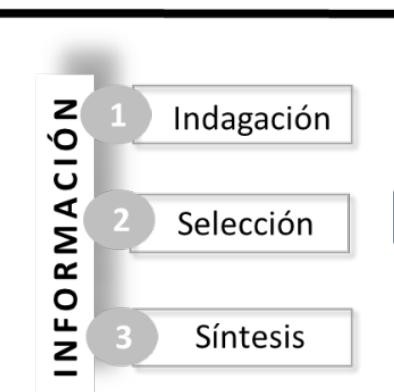

Recursos

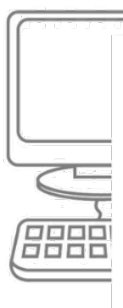

Easel.ly

Piktochart

Canva

Create.ly

Infogram

Powerpoint

\section{Producto}

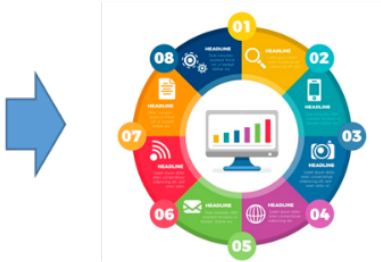

Fig 1. Esquema con los pasos seguidos por los alumnos para la elaboración de las infografias.

En la siguiente sesión dedicada a esta actividad, se repartió a cada alumno una copia de cada una de las 12 infografías junto con un cuestionario (Figura 2) que debían contestar de forma individual valiéndose únicamente de la información encontrada en ellas, con la idea de valorar si la infografía estaba correctamente realizada. Una vez finalizado el cuestionario, se recogió y la profesora explicó las características de cada dieta con más profundidad y cuáles eran las respuestas correctas, para evitar cualquier queja cognitiva. Se consiguió crear un debate en el que participaron la mayor parte de los alumnos. Hay que señalar que en esta actividad se valoró la participación pero no las respuestas acertadas en el cuestionario, ni la calidad de las infografías. Sí se dio la oportunidad a los alumnos de votar a las mejores infografías. El ránking con las tres más votadas se publicó en el aula virtual de la asignatura. Para evaluar el éxito del proyecto, al finalizar la sesión se pasó un cuestionario a los alumnos para que indicaran su grado de satisfacción con la misma.

\section{Resultados}

\subsection{Infografías de las dietas más comunes en occidente}

Cada grupo de alumnos presento una de las 12 infografías sobre las dietas de estudio (ver Figura 3). La herramienta más utilizada para crearlas fue el Infogram, seguido del Powerpoint y del Piktochart. Los resultados obtenidos fueron dispares. Algunas de ellas sí que se ajustaban a las características de las infografías, otras sin embargo utilizaban demasiado texto escrito. El contenido de las infografías era correcto en todos los casos. 
Los alumnos votaron los trabajos presentados valorando si se ajustaban a las características propias de una infografía y en función de la cantidad de la información que les habían aportado. En la Tabla 1 aparecen ordenadas las infografías de mejor a peor valoradas.

Tabla 1. Valoración de las infografias por parte de los estudiantes

\begin{tabular}{|c|c|}
\hline Posición & Dieta \\
\hline 1 & Nórdica \\
\hline 2 & DASH \\
\hline 3 & Atkins \\
\hline 4 & Macrobiótica \\
\hline 5 & Paleo \\
\hline 6 & Flexitariana \\
\hline 7 & Ayuno intermit \\
\hline 8 & Disociada \\
\hline 9 & Dukan \\
\hline 10 & MIND \\
\hline 11 & DETOX \\
\hline 12 & Raw-food \\
\hline
\end{tabular}
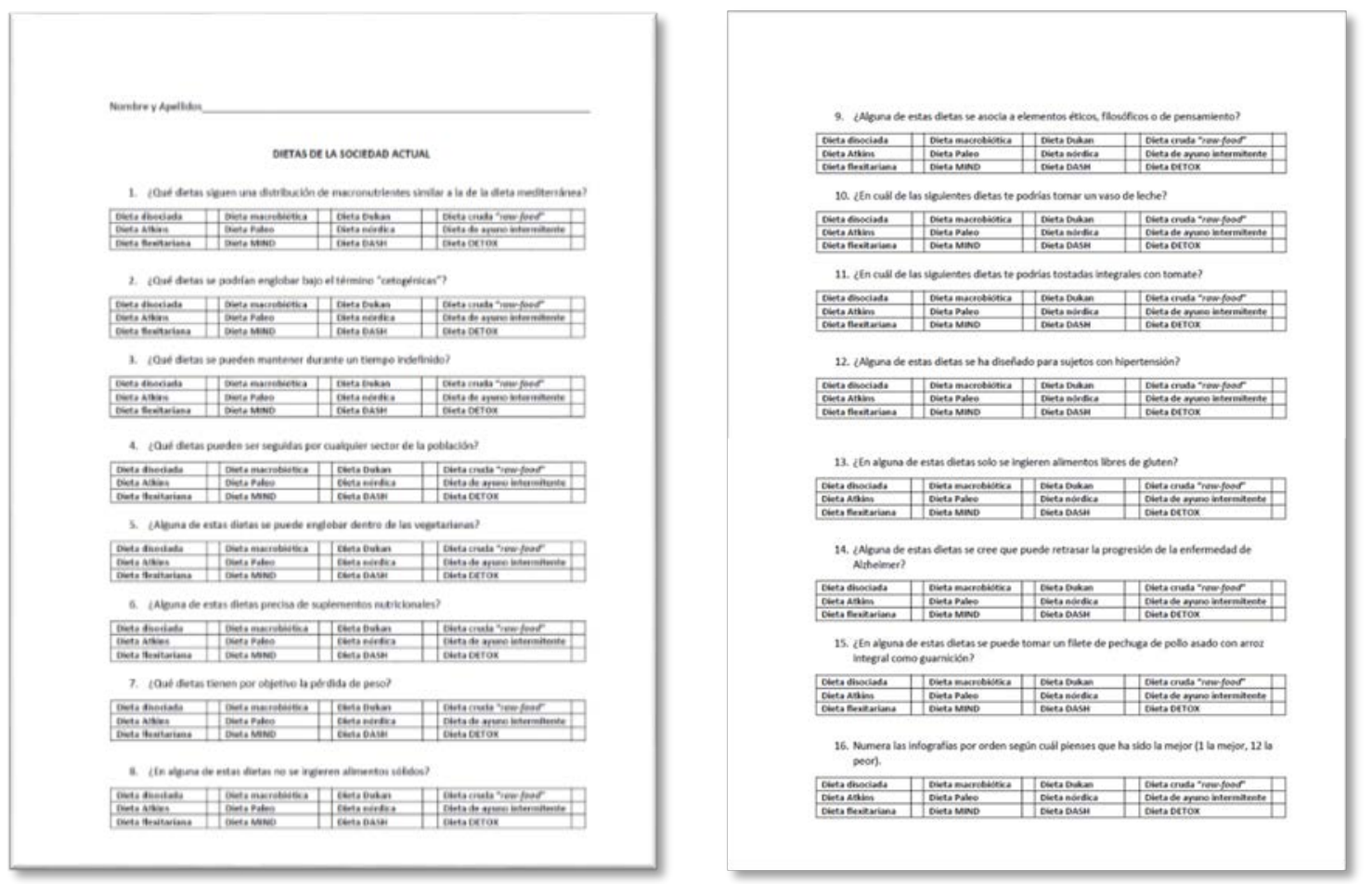

Fig 2. Imagen del cuestionario que los alumnos debian contestar utilizando únicamente las infografías realizadas por ellos como fuente de información. 


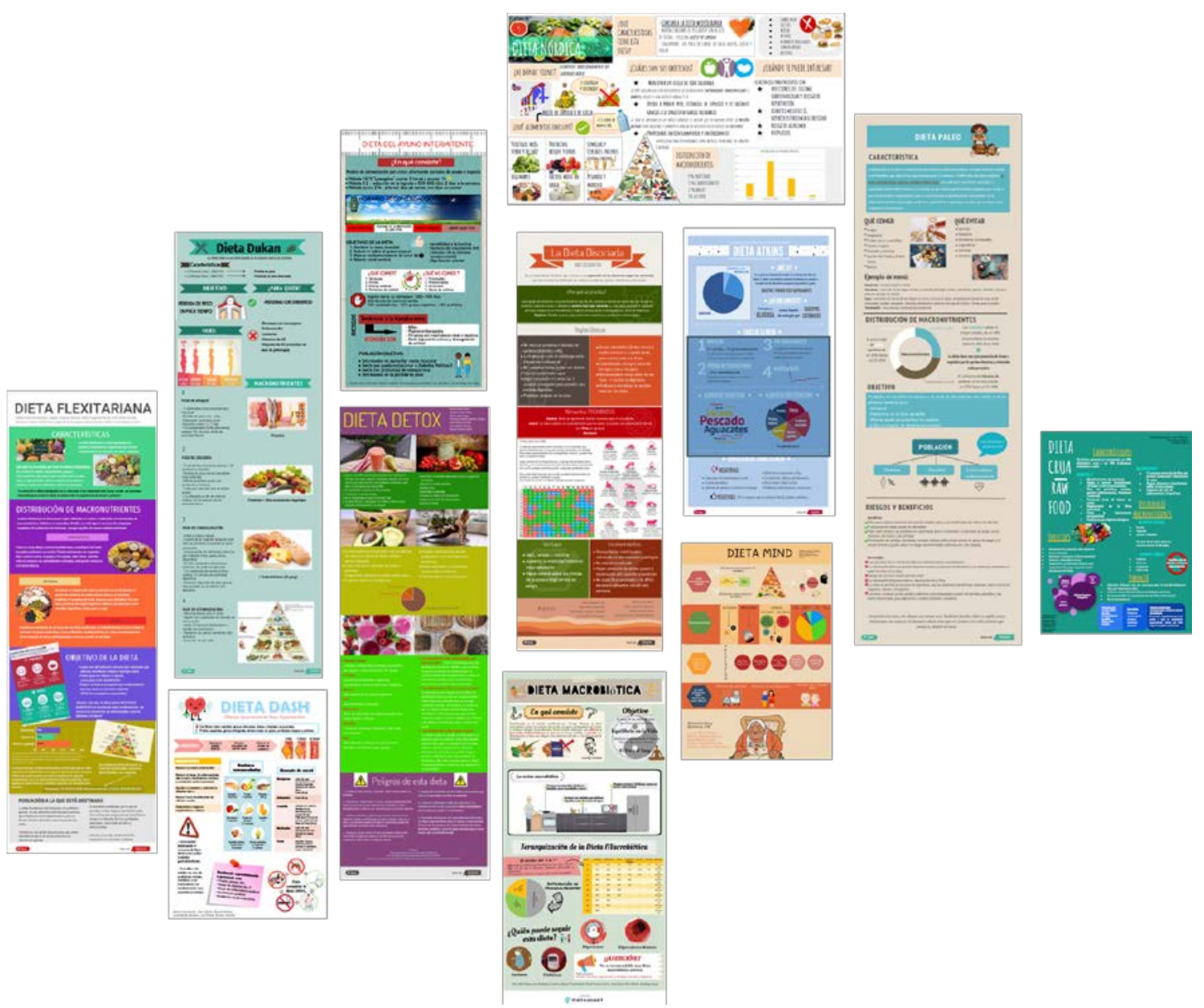

Fig 3. Infografias elaboradas por los alumnos.

\subsection{Resultados del aprendizaje}

Como se puede observar en las Figuras 4-6, aunque la mayor parte del alumnado fue capaz de identificar las respuestas correctas al cuestionario sobre las dietas que debían contestar utilizando únicamente las infografías, en algunos casos fue necesario completar, aclarar o matizar algunas características de las mismas. Por ejemplo en la Figura 4 se puede ver que muchos alumnos no han considerado que la dietas de ayuno intermitente tengan una distribución de macronutrientes equivalente a la de la dieta mediterránea, cuando si es así. No cambia el porcentaje de ingesta de macronutrientes sino el periodo durante el que se puede realizar dicha ingesta. Precisamente para evitar que al alumnado le quedaran ambigüedades sobre los contenidos, la última parte de la misma, la puesta en común y explicación final es esencial. 


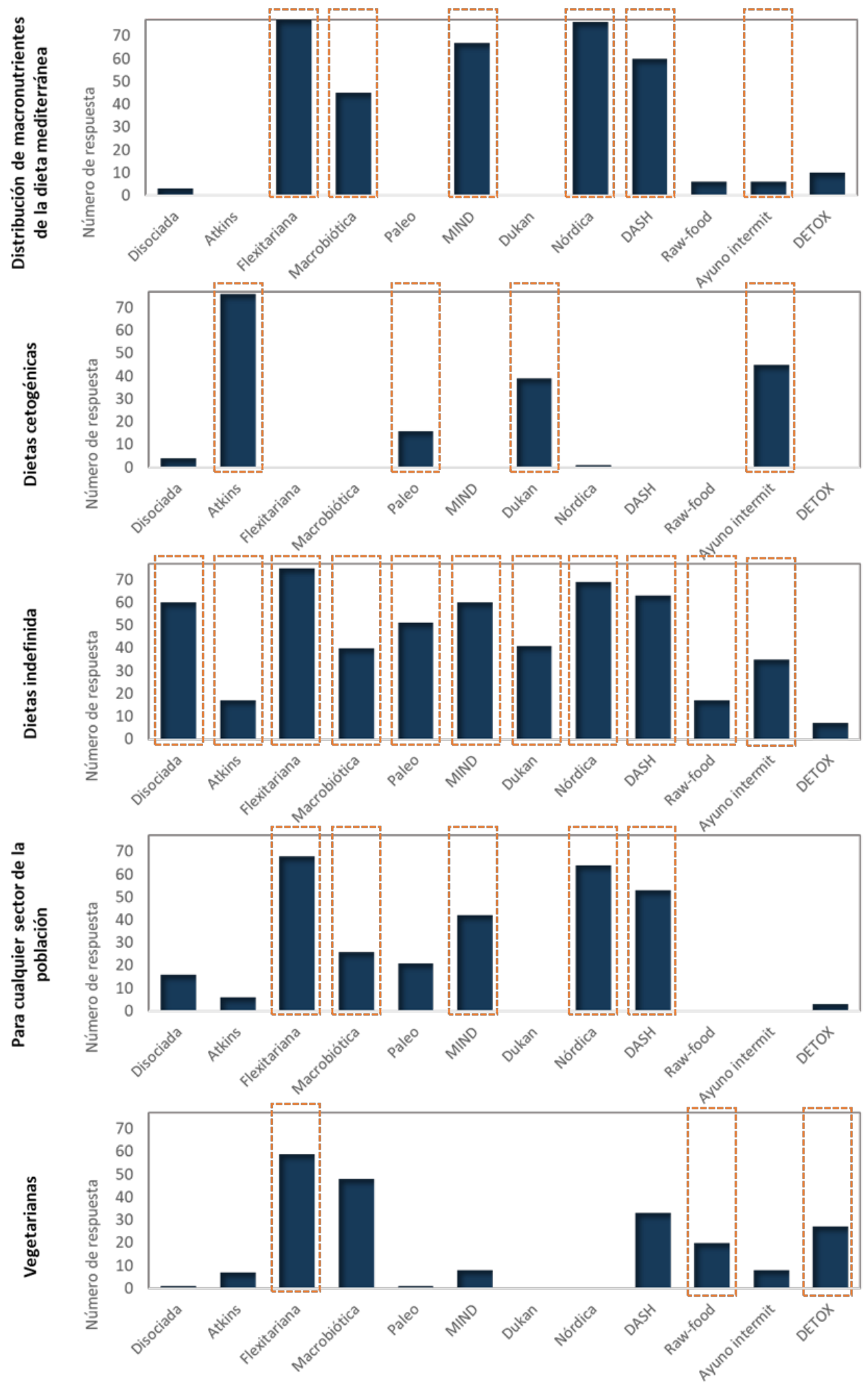

Fig 4. Respuestas de los alumnos al cuestionario sobre las características de las dietas estudiadas (parte 1). La única herramienta que han utilizado para contestar son las infografias de las dietas que han elaborado en grupos. El número total de alumnos que participaron en la sesión fue de 74. Con el punteado naranja se representa el patrón que se debeía haber obtenido si todos los alumnos hubiesen contestado correctamente el cuestionario. 

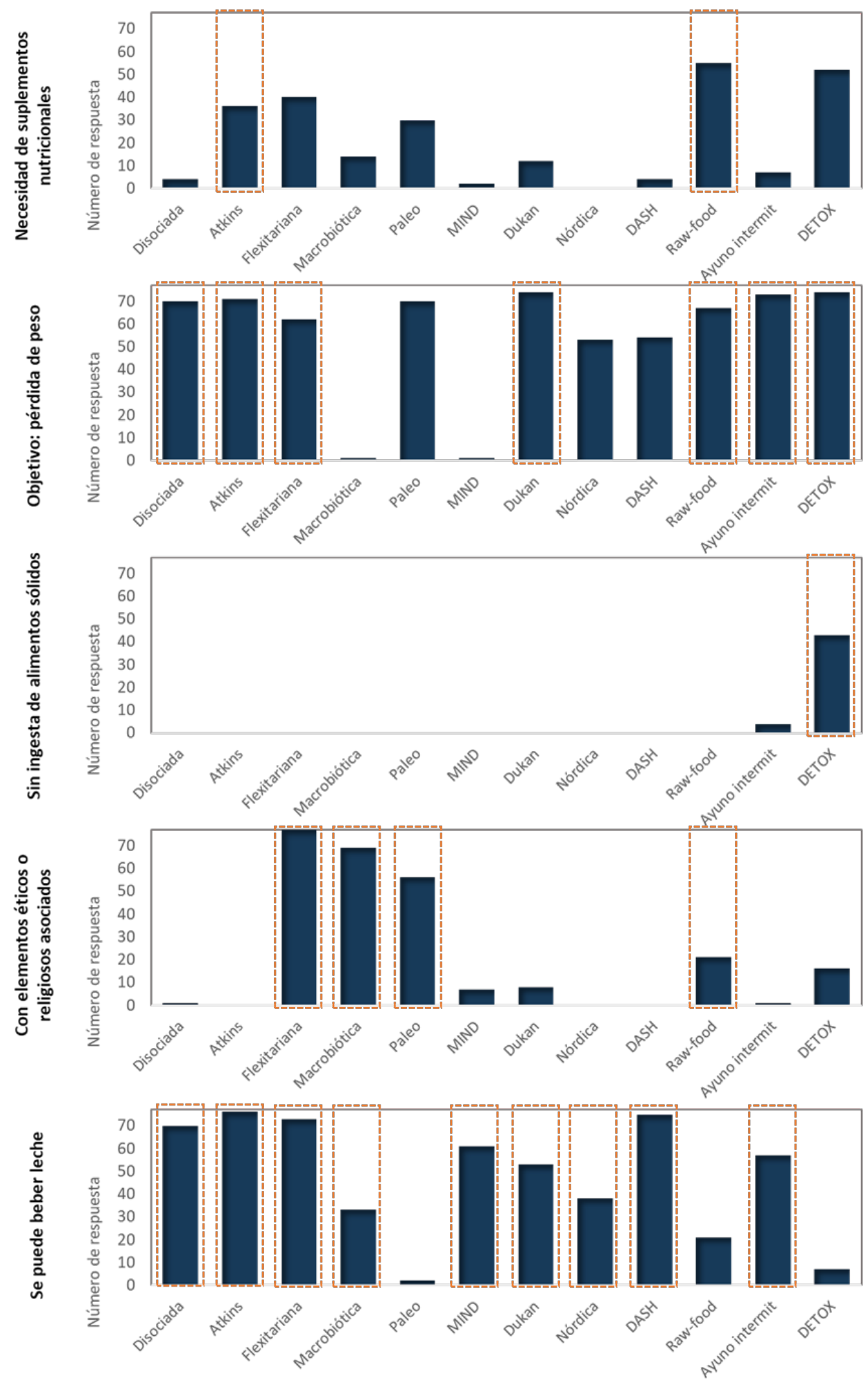

Fig 5. Respuestas de los alumnos al cuestionario sobre las características de las dietas estudiadas (parte 2). La única herramienta que han utilizado para contestar son las infografias de las dietas que han elaborado en grupos. El número total de alumnos que participaron en la sesión fue de 74. Con el punteado naranja se representa el patrón que se debeía haber obtenido si todos los alumnos hubiesen contestado correctamente el cuestionario. 

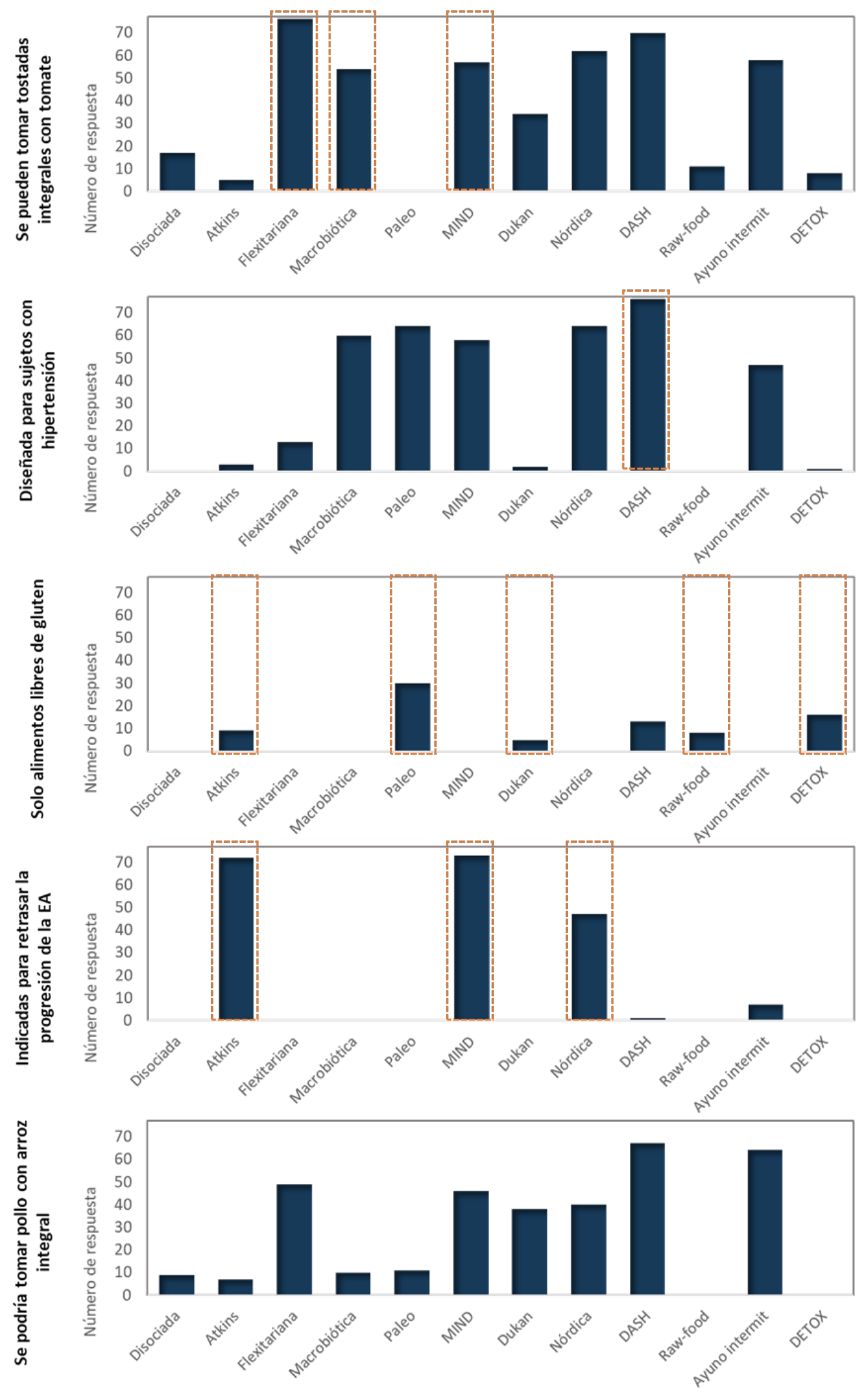

Fig 6. Respuestas de los alumnos al cuestionario sobre las características de las dietas estudiadas (parte 2). La única herramienta que han utilizado para contestar son las infografias de las dietas que han elaborado en grupos. El número total de alumnos que participaron en la sesión fue de 74. Con el punteado naranja se representa el patrón que se debeía haber obtenido si todos los alumnos hubiesen contestado correctamente el cuestionario. 


\subsection{Valoración de la actividad}

La actividad fue en general bien valorada por la mayor parte de los alumnos como se puede desprender se sus respuestas al cuestionario anónimo de valoración de la actividad. A la pregunta "Con esta actividad ¿crees que ha aumentado tu conocimiento sobre los distintos tipos de dietas que se siguen actualmente?" la mayoría de los estudiantes han contestado que están de acuerdo o toalmente de acuerdo (Ver Figura 7)

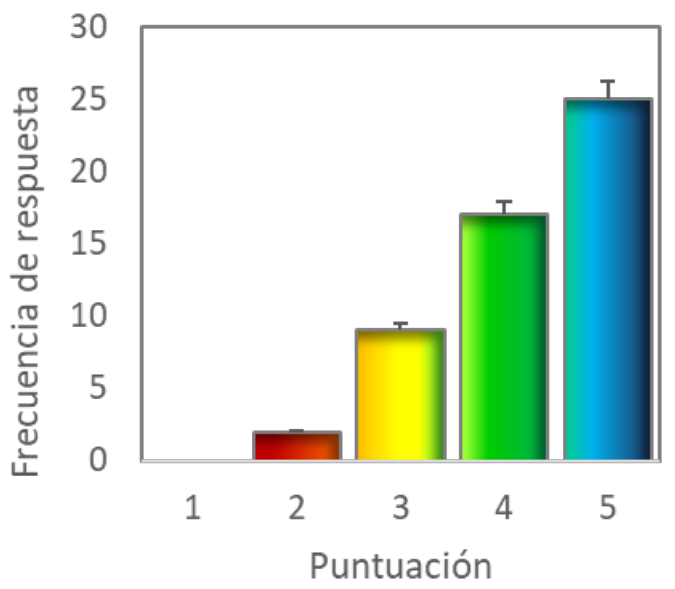

Fig 7. Valoración de los estudiantes a la pregunta ¿Consideras que que gracias a los debates en los que has participado has aprendido? 1: Totalmente de desacuerdo. 5: Totalmente de acuerdo. Se muestra la frecuencia de las respuestas y un error estándar asumido del $5 \%$ a la hora de rellenar/contabilizar las encuestas.

En la Figura 8 aparece la valoración que hacen los estudiantes sobre si la actividad mejora de sus habilidades digitales. Según los resultados para esa pregunta, los alumnos no parecen percibir gran mejora en dichas habilidades. La mayor parte de los alumnos han contestado con notas iguales o inferiores a 3 . Probablemente sea debido a que ya poseían esas habilidades digitales, ya que hoy en día es muy común que todos ellos tengan cuenta en plataformas como Pinterest o Instagram.

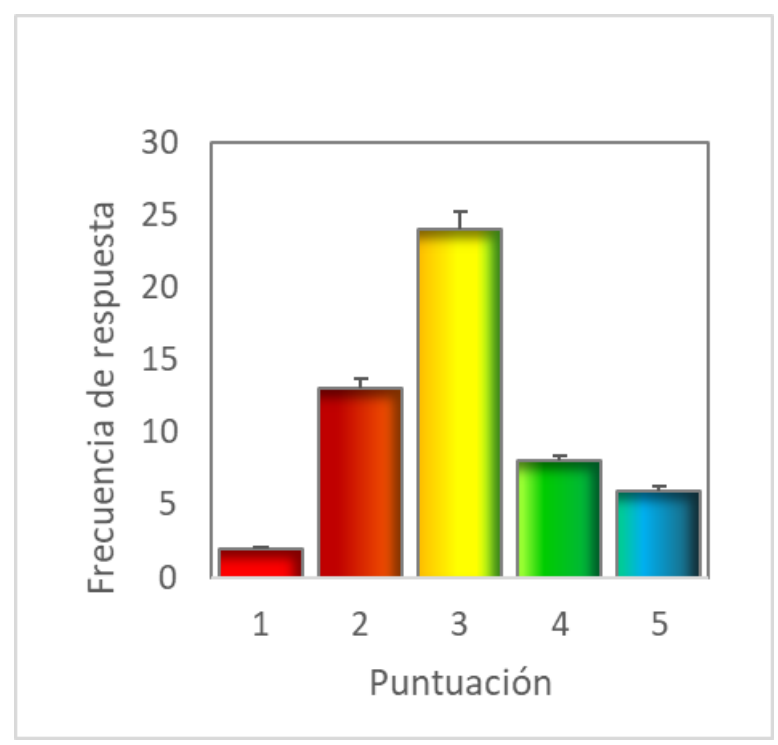

Fig 8. Valoración de los estudiantes a la pregunta “Con esta actividad ¿crees que han mejorado tus habilidades digitales?” 1: Totalmente de desacuerdo. 5: Totalmente de acuerdo. Se muestra la frecuencia de las respuestas y un error estándar asumido del $5 \%$ a la hora de rellenar/contabilizar las encuestas.

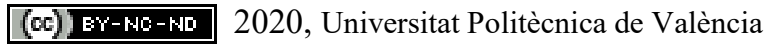

Congreso In-Red (2020) 
Los resultados muestran que los alumnos sí prefieren este tipo de actividad a una clase magistral que trate los mismos contenidos (ver Figura 9) y que sí que consideran que la infografía es una herramienta útil como metodología docente (ver Figura 10) que podrán utilizar en presentaciones y trabajos de otras asignaturas de la carrera (ver Figura 11).

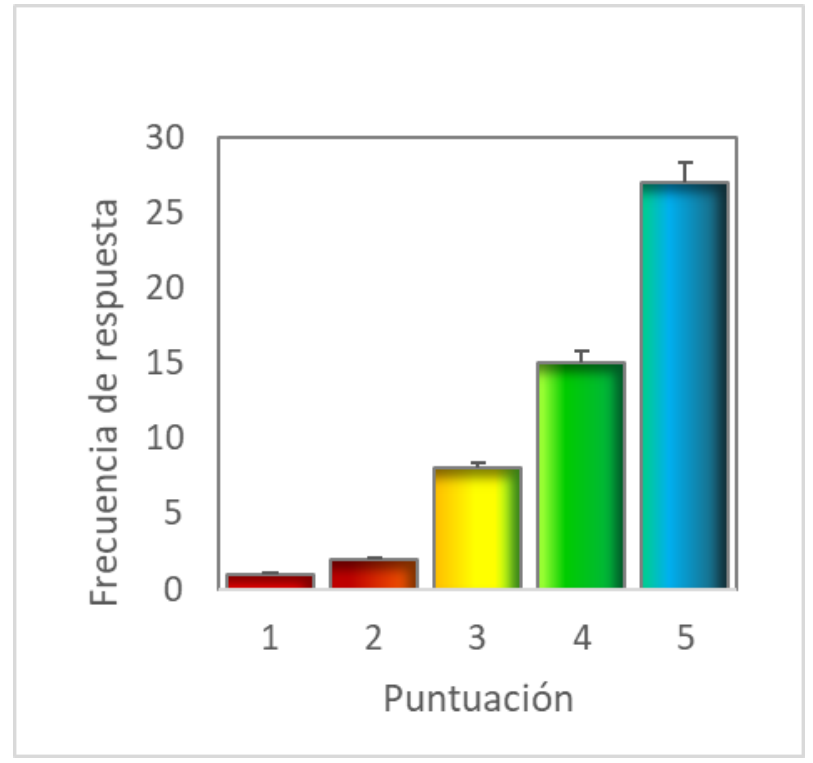

Fig 9. Valoración de los estudiantes a la pregunta “¿Prefieres este tipo de actividad a una clase magistral que trate los mismos contenidos?"1: Totalmente de desacuerdo. 5: Totalmente de acuerdo. Se muestra la frecuencia de las respuestas y un error estándar asumido del $5 \%$ a la hora de rellenar/contabilizar las encuestas.

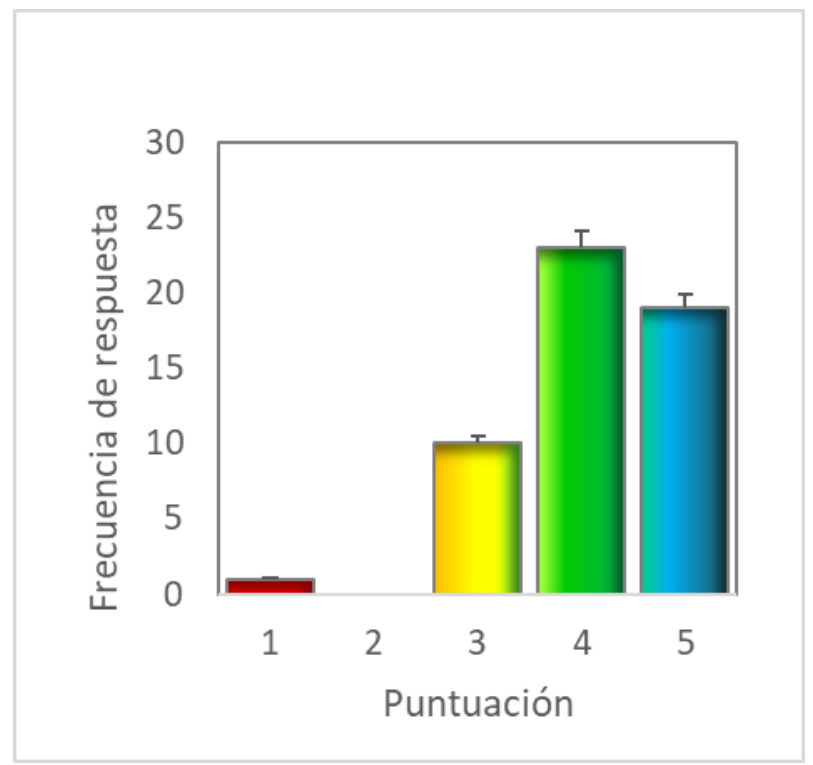

Fig 10. Valoración de los estudiantes a la pregunta “¿Crees que la infografia es una herramienta útil como metodología docente?" 1: Totalmente de desacuerdo. 5: Totalmente de acuerdo. Se muestra la frecuencia de las respuestas y un error estándar asumido del $5 \%$ a la hora de rellenar/contabilizar las encuestas. 


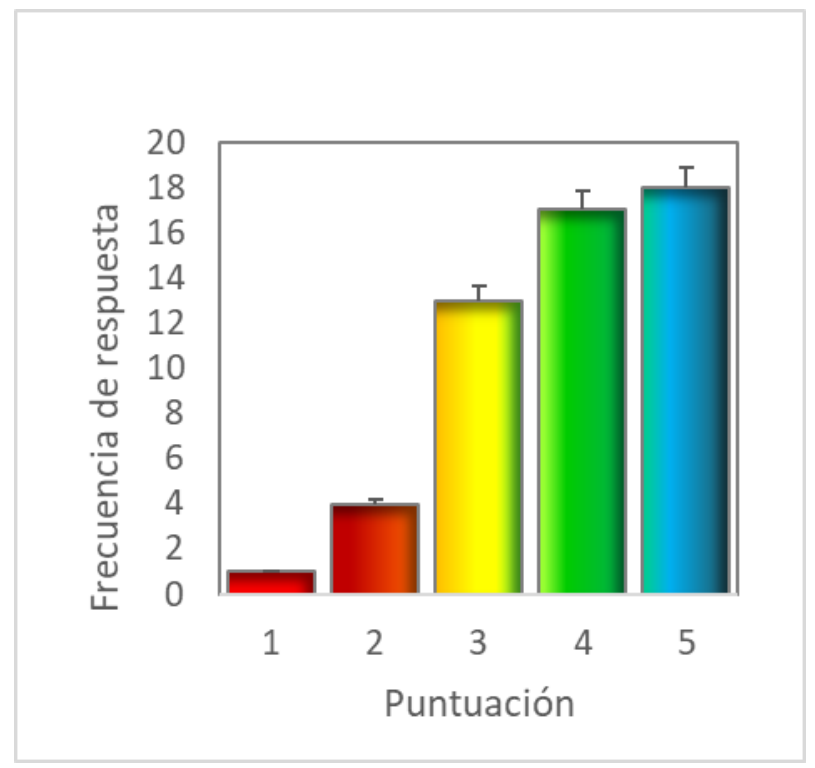

Fig 11. Valoración de los estudiantes a la pregunta “¿Crees que crear infografías te será útil para presentaciones o trabajos de otras asignaturas de la carrera? 1: Totalmente de desacuerdo. 5: Totalmente de acuerdo. Se muestra la frecuencia de las respuestas y un error estándar asumido del 5\% a la hora de rellenar/contabilizar las encuestas.

También se pidió a los alumnos su opinión sobre si esta actividad les ayudaba a adquirir alguna de las competencias de su titulación. Para ello contestaron a las siguientes preguntas: “¿Crees que esta actividad contribuye a mejorar tu capacidad de transmitir información?” “¿Crees que esta actividad contribuye a mejorar tu capacidad para trabajar en equipo?” ¿Crees que esta actividad contribuye a mejorar tu capacidad crítica y autocrítica?” “Crees que esta actividad contribuye a mejorar tu creatividad?” (ver Figuras 12-16). En general la impresión de los estudiantes es que la actividad sí que ayuda a mejorar dichas competencias.

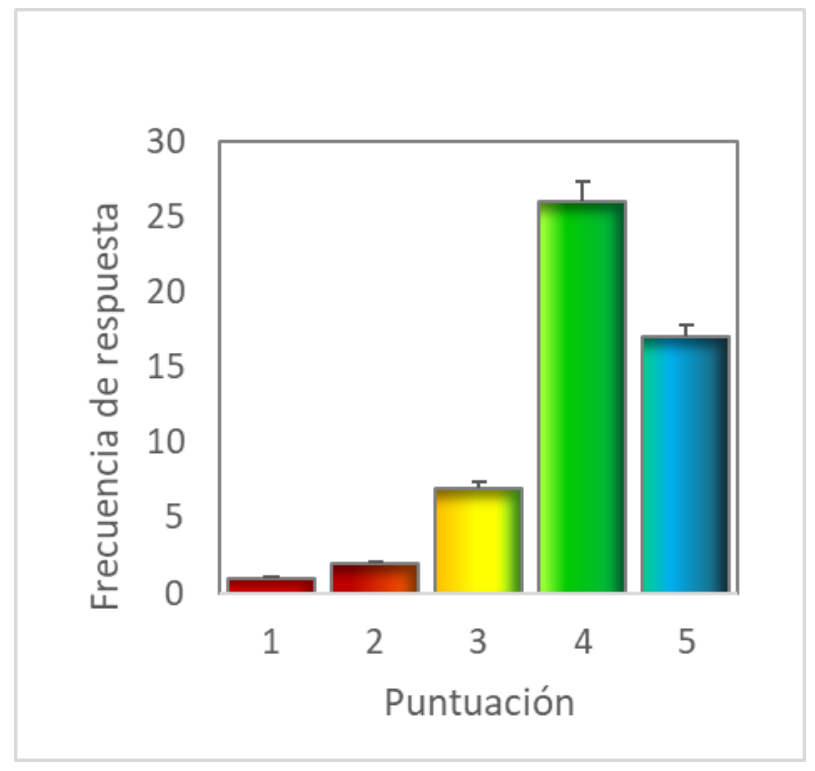

Fig 12. Valoración de los estudiantes a la pregunta ¿Crees que esta actividad contribuye a mejorar tu capacidad de reunir e interpretar datos relevantes? 1: Totalmente de desacuerdo. 5: Totalmente de acuerdo. y un error estándar del 5\% a la hora de rellenar las encuestas. Se muestra la frecuencia de las respuestas y un error estándar asumido del 5\% a la hora de rellenar/contabilizar las encuestas.

(c) EY-NC-ND 2020, Universitat Politècnica de València

Congreso In-Red (2020) 


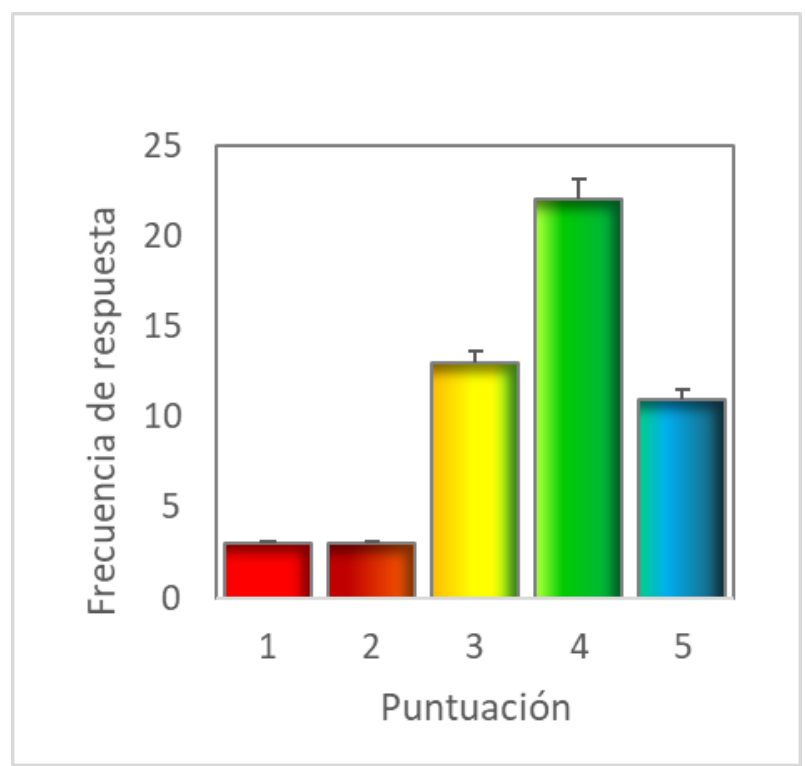

Fig 13. Valoración de los estudiantes a la pregunta “¿Crees que esta actividad contribuye a mejorar tu capacidad de transmitir información?”. 1: Totalmente de desacuerdo. 5: Totalmente de acuerdo. Se muestra la frecuencia de las respuestas y un error estándar asumido del $5 \%$ a la hora de rellenar/contabilizar las encuestas.

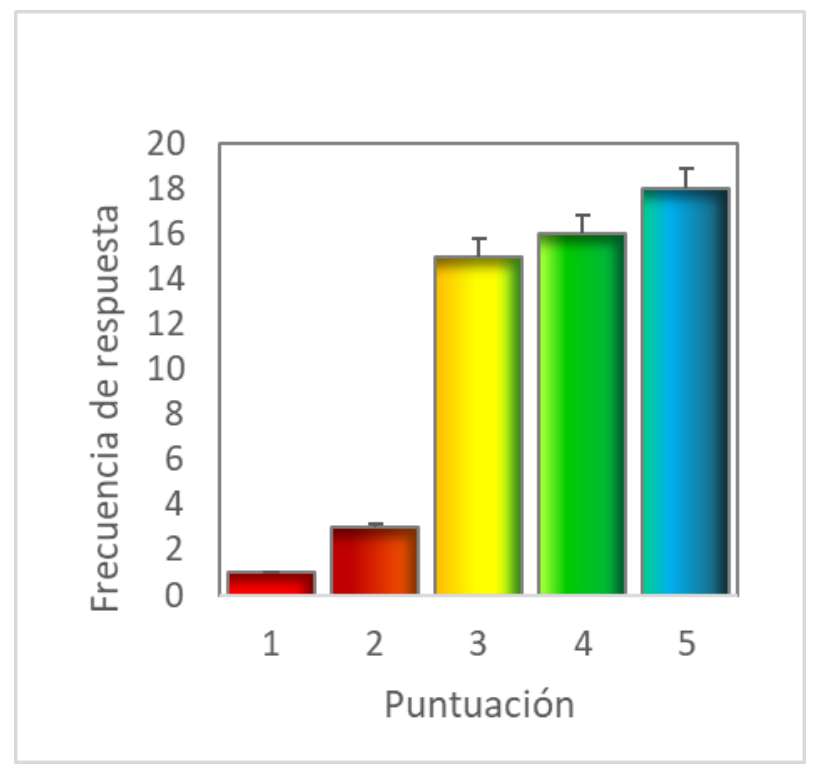

Fig 14. Valoración de los estudiantes a la pregunta “¿Crees que esta actividad contribuye a mejorar tu capacidad critica y autocrítica?”. 1: Totalmente de desacuerdo. 5: Totalmente de acuerdo. Se muestra la frecuencia de las respuestas y un error estándar asumido del 5\% a la hora de rellenar/contabilizar las encuestas. 


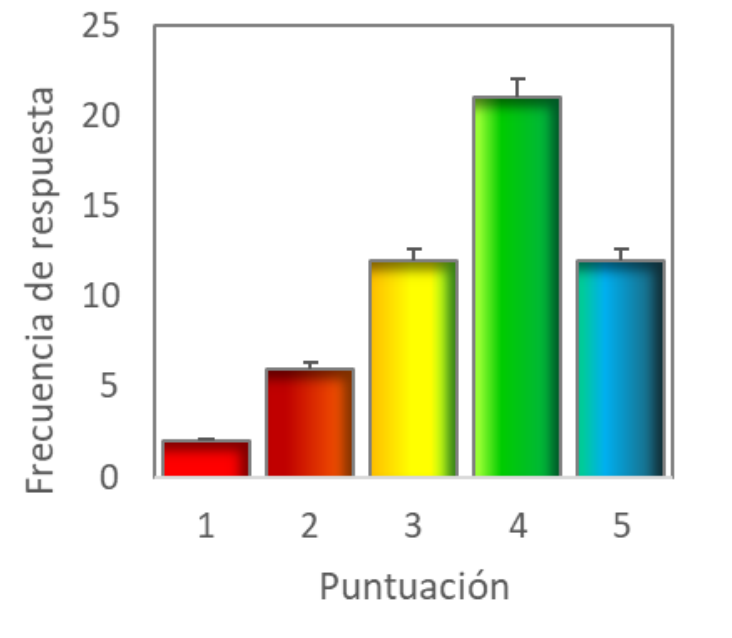

Fig 15. Valoración de los estudiantes a la pregunta “¿Crees que esta actividad contribuye a mejorar tu capacidad crítica y autocrítica?". 1: Totalmente de desacuerdo. 5: Totalmente de acuerdo. Se muestra la frecuencia de las respuestas y un error estándar asumido del $5 \%$ a la hora de rellenar/contabilizar las encuestas.

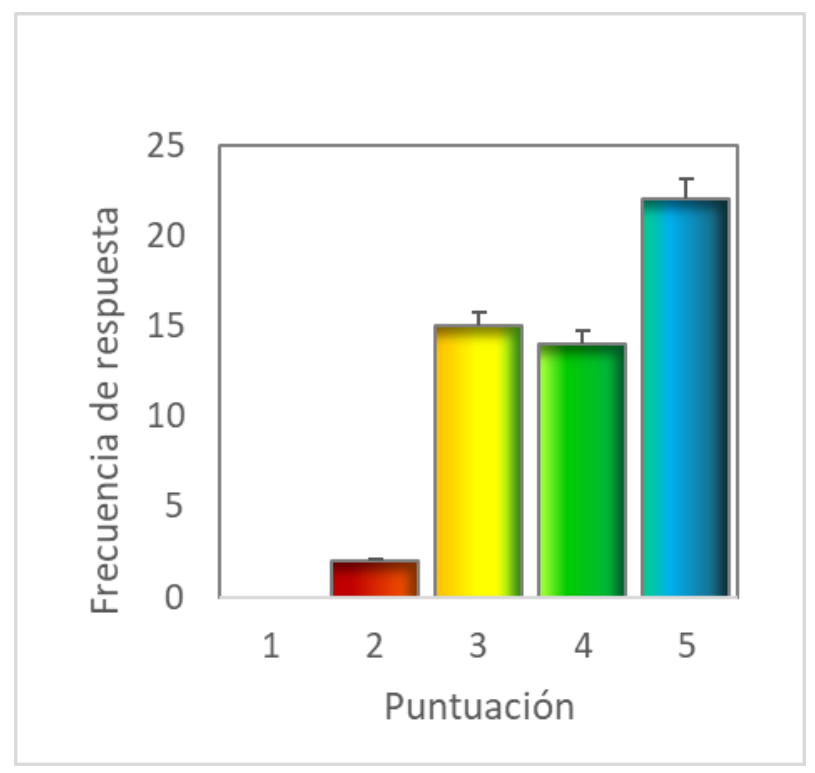

Fig 16. Valoración de los estudiantes a la pregunta “¿Crees que esta actividad contribuye a mejorar tu creatividad? 1: Totalmente de desacuerdo. 5: Totalmente de acuerdo. Se muestra la frecuencia de las respuestas y un error estándar asumido del $5 \%$ a la hora de rellenar/contabilizar las encuestas.

Los alumnos también han valorado positivamente la parte lúdica de la actividad. Como muestra la Figura 17 , prácticamente todos la encuentran entretenida. 


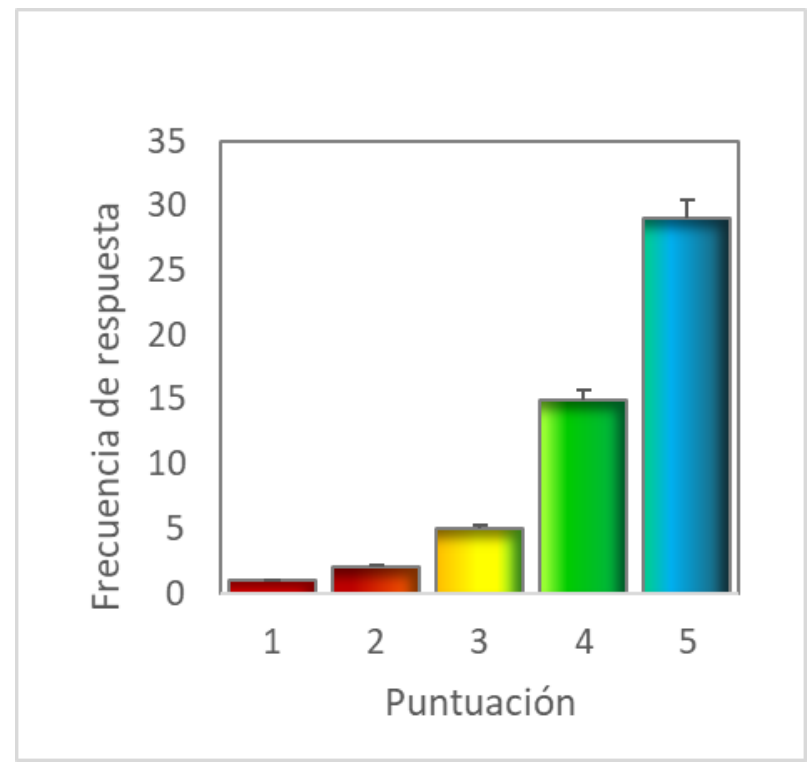

Fig 17. Valoración de los estudiantes a la pregunta “¿La actividad te ha resultado entretenida?” 1: Totalmente de desacuerdo. 5: Totalmente de acuerdo. Se muestra la frecuencia de las respuestas y un error estándar asumido del $5 \%$ a la hora de rellenar/contabilizar las encuestas.

Por último, los alumnos valoran muy positivamente la actividad desde un punto de vista global.

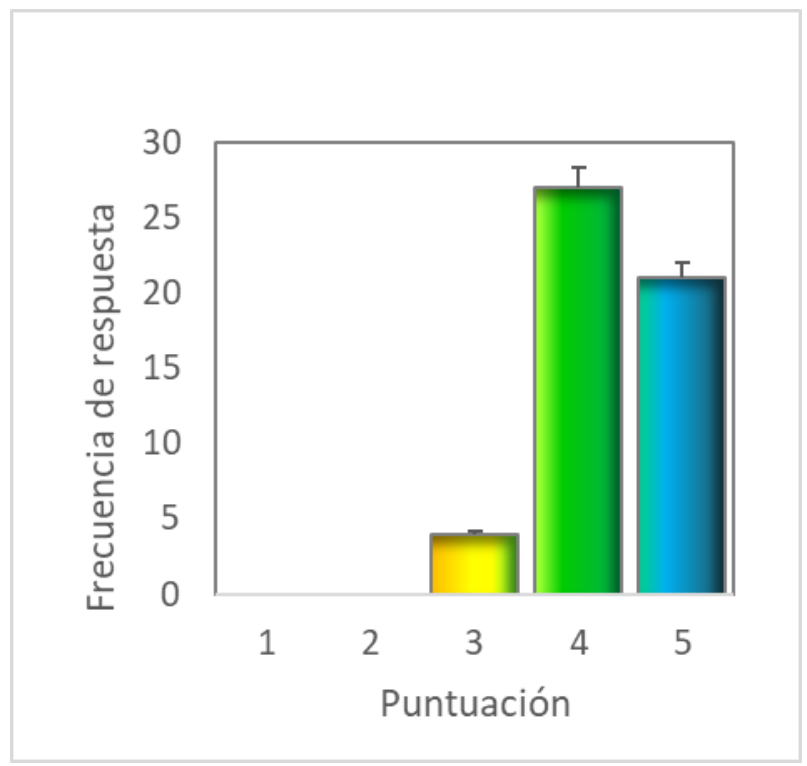

Fig 18. Valoración del grado de satisfacción global de los estudiantes con la actividad. 1: Totalmente de desacuerdo. 5: Totalmente de acuerdo. Se muestra la frecuencia de las respuestas y un error estándar asumido del 5\% a la hora de rellenar/contabilizar las encuestas. 


\section{Conclusiones}

El uso de infografías, realizadas por los mismos alumnos, para abordar el tema de las dietas de moda en la sociedad occidental actual es una alternativa a la clase magistral que parece funcionar bien. Por una parte, gracias a la utilización de los cuestionarios sobre dichas dietas, se puede comprobar que no existe queja cognitiva y, en caso de detectarse alguna, corregirla. Por otro lado, se profundiza más en la materia de la asignatura, permitiendo analizar un número mayor de dietas. Además la actividad es bien valorada por los alumnos, que consideran que con ella han mejorado sus conocimientos sobre la materia, se han entretenido, han mejorado su capacidad de búsqueda y síntesis de información, así como su capacidad de autocrítica. También opinan que la infografía es una herramienta que podrán utilizar en presentaciones de otras asignaturas de la materia. Por tanto, a la luz de los resultados obtenidos se puede concluir que la implementación del uso de infografías como herramienta docente en el grado de Medicina, podría ser interesante y bien acogido por los estudiantes. .

\section{Referencias}

ACUÑA M. (2018). El poder de la Infografia en el aprendizaje”. EVirtualplus.

$<$ https://www.evirtualplus.com/infografia-en-el-aprendizaje> [Consulta: 20 de marzo de 2020].

BRADSHAW MJ, PORTER S. (2017). Infographics: A New Tool for the Nursing Classroom. en Nurse Educ. Vol.42, issue 2, p. 57-59.

GARCÍA EM. (2016). Uso didáctico de las infografias. en Espiral Cuadernos Del Profesorado. vol. 7, issue 14, p. $37-44$.

MCCRORIE AD, DONNELLY C, MCGLADE KJ. (2016). Infographics: Healthcare Communication for the Digital Age. en Ulster Med J. vol. 85, issue 2, p. 71-75.

RONEY AGUIRRE C, MENJÍVAR VALENCIA E, MORALES HL. (2015). Elaboración de infografias: hacia el desarrollo de competencias del siglo XXI, vol. 15, p. 23-37.

PROVVIDENZA CF, HARTMAN LR, CARMICHAEL J, REED N. (2019). Does a picture speak louder than words? The role of infographics as a concussion education strategy. en J Vis Commun Med. Vol. 42, issue 3, p.102113. 